\title{
Tungsten and barium transport in the internal plasma of hollow cathodes
}

\author{
James E. Polk, ${ }^{1, a)}$ Ioannis G. Mikellides, ${ }^{1}$ Ira Katz, ${ }^{1}$ and Angela M. Capece ${ }^{2}$ \\ ${ }^{1}$ Jet Propulsion Laboratory, California Institute of Technology, 4800 Oak Grove Drive, Pasadena, \\ California 91109, USA \\ ${ }^{2}$ California Institute of Technology, 1200 E. California Blvd., Pasadena, California 91125, USA
}

(Received 18 September 2008; accepted 2 March 2009; published online 1 June 2009)

\begin{abstract}
The effect of tungsten erosion, transport, and redeposition on the operation of dispenser hollow cathodes was investigated in detailed examinations of the discharge cathode inserts from $8200 \mathrm{~h}$ and $30352 \mathrm{~h}$ ion engine wear tests. Erosion and subsequent redeposition of tungsten in the electron emission zone at the downstream end of the insert reduce the porosity of the tungsten matrix, preventing the flow of barium from the interior. This inhibits the interfacial reactions of the barium-calcium-aluminate impregnant with the tungsten in the pores. A numerical model of barium transport in the internal xenon discharge plasma shows that the barium required to reduce the work function in the emission zone can be supplied from upstream through the gas phase. Barium that flows out of the pores of the tungsten insert is rapidly ionized in the xenon discharge and pushed back to the emitter surface by the electric field and drag from the xenon ion flow. This barium ion flux is sufficient to maintain a barium surface coverage at the downstream end greater than 0.6, even if local barium production at that point is inhibited by tungsten deposits. The model also shows that the neutral barium pressure exceeds the equilibrium vapor pressure of the impregnant decomposition reaction over much of the insert length, so the reactions are suppressed. Only a small region upstream of the zone blocked by tungsten deposits is active and supplies the required barium. These results indicate that hollow cathode failure models based on barium depletion rates in vacuum dispenser cathodes are very conservative. () 2009 American Institute of Physics.
\end{abstract}

[DOI: $10.1063 / 1.3111970]$

\section{INTRODUCTION}

Electric propulsion offers significant trip time and/or mass benefits for a wide range of deep space missions, ${ }^{1}$ but the thrusters in these systems are required to operate for tens of thousands of hours to provide the necessary total impulses. This requirement places extraordinary demands on component reliability and makes thruster qualification difficult. Hollow cathode electron sources are key life-limiting components in these engines and this paper describes experiments and modeling aimed at understanding cathode failures due to material transport.

State-of-the-art hollow cathodes consist of a porous tungsten tube (the "insert"), which is contained in a refractory metal cathode tube with an orifice plate on the downstream end. A small fraction of the thruster propellant is injected through the hollow cathode and the orifice serves to increase the internal pressure in the insert region. Electron emission from the inner surface maintains an internal plasma that heats the insert to the required operating temperature and helps conduct the current into the main discharge. A heater surrounding the cathode is used to preheat it prior to ignition.

A low emitter operating temperature is achieved by maintaining a layer of adsorbed oxygen and barium atoms that lowers the surface work function. In state-of-the-art impregnated cathodes, $\mathrm{Ba}$ and $\mathrm{BaO}$ are supplied by barium calcium aluminate source material (the "impregnant") incorporated in the pores of the tungsten. Gaseous $\mathrm{Ba}$ and $\mathrm{BaO}$ are

${ }^{a)}$ Electronic mail: james.e.polk@jpl.nasa.gov. released in interfacial reactions between the tungsten matrix and the impregnant, producing a temperature-dependent vapor pressure of these species inside the pores. $\mathrm{Ba}$ and $\mathrm{BaO}$ then migrate to the surface by Knudsen flow and surface diffusion on the pore walls. In vacuum dispenser cathodes $\mathrm{Ba}$ adsorbates lost by evaporation are replenished by $\mathrm{Ba}$ and $\mathrm{BaO}$ through surface diffusion from the pores and most of the vapor flow from the pores is lost. In gas discharges, the vapor in the discharge may be transported back to the cathode surface, offering another path for replenishing Ba lost by desorption.

Near the surface, products of the impregnant reactions escape to the exterior through the pores, allowing the reactions to proceed to completion. Eventually, relatively stable tungstates are produced and production of volatile species in that region ceases. The reaction front proceeds into the insert as reactants nearer the surface are consumed and the porosity of the impregnant increases due to loss of volatile materials. The region behind the reaction front is depleted in barium and the depth of this depletion layer has been characterized in some cathode tests ${ }^{2}$ as an indicator of remaining useful life.

Hollow cathodes are subject to several potential failure mechanisms. External erosion by high energy ions created downstream of the orifice has been observed in a number of tests $^{3-5}$ and is the focus of experiments and modeling discussed elsewhere. ${ }^{6,7}$ Damage to the emitting surface due to poisoning by reactive gases or buildup of bulk tungstate layers due to reactive impurities is another potential failure mode. ${ }^{8-10}$ 
Insert life is ultimately limited by the barium supply in the impregnant. When the barium supply rate from the interior or from the gas phase drops below the rate at which adsorbed atoms are lost from the surface by desorption, the surface coverage drops and the work function rises. ${ }^{11}$ The cathode eventually becomes impossible to ignite or cannot be heated to the temperatures needed for the required electron current density.

Finally, tungsten transport has been observed in many extended hollow cathode tests. ${ }^{3,5,11,12}$ Impregnated cathode temperatures are not high enough to cause significant evaporation of the tungsten matrix, so these processes are undoubtedly due to formation and subsequent dissociation of volatile tungsten compounds. No failures have been attributed to this mechanism, but it could potentially lead to cathode failure. Reduction in the cathode orifice diameter by deposition of tungsten eroded from internal surfaces could increase the operating temperature or prevent cathode ignition and deposits of tungsten on the emitter surface may limit barium flow through the tungsten matrix.

The lack of understanding of the chemical and mass transport processes in hollow cathodes prevented development of useful models for these failure mechanisms. This paper attempts to answer two fundamental questions as a first step to developing the required insight. First, does erosion, transport, and deposition of tungsten in the insert region affect hollow cathode function? Second, how does gas phase transport of barium affect the operation of the cathode? These two questions turned out to be intimately linked.

The first question has been approached by a detailed examination of the cathode inserts used in several long duration ion engine wear tests. The $8200 \mathrm{~h}$ life demonstration test (LDT) was performed with a $30 \mathrm{~cm}$ engineering model thruster similar to that flown on the Deep Space 1 (DS1) mission ${ }^{13,14}$ under the NASA solar electric propulsion technology applications readiness (NSTAR) program. ${ }^{3}$ A subsequent extended life test (ELT) was performed with the DS1 flight spare NSTAR thruster, ultimately accumulating 30352 $\mathrm{h}$ of operation at several different throttle levels. ${ }^{4}$ Both tests were voluntarily terminated with the discharge and neutralizer cathodes exhibiting no evidence of performance degradation. The examination of these cathodes described here extends initial evaluations reported earlier ${ }^{3,5}$ and focused specifically on material transport. The objectives were to identify erosion and deposition sites and to determine if these processes modified or have the potential to impact the function of the insert as an electron emitter.

The second question was addressed by developing a model of barium transport in the gas phase. This model leverages significant work done in modeling the internal xenon gas discharge. The objectives were to study how barium enters the discharge, flows through the plasma, and is redeposited on the emitter surface, and how that affects the cathode work function.

\section{EXPERIMENTAL CHARACTERIZATION OF MATERIAL TRANSPORT PROCESSES}

In many long-duration hollow cathode tests, restructuring of the tungsten insert and orifice plate has been observed. ${ }^{3,5,11,12}$ This typically involves erosion in the emission zone and upstream face of the orifice plate, formation of tungsten crystals at the downstream end of the emitter, and deposition of tungsten, often in the form of large crystals on the orifice plate and within the orifice. In some cases, films of barium compounds have been observed on the emitter surface and tungsten has been found with barium oxide in deposits between the emitter and the cathode tube. ${ }^{12}$

A link with reactive gases is implied by Sarver-Verhey's observation of extreme examples of these erosion and deposition phenomena in a series of cathodes that had been operated with leaky gas systems. ${ }^{12} \mathrm{He}$ speculated that tungsten transport was due to the formation of volatile tungsten compounds, which were subsequently dissociated, leaving pure tungsten deposits. The deposition of barium compounds on the emitter surface also appears to be related to the presence of oxidizing impurities. ${ }^{9,10}$ However, the flow dynamics of barium and tungsten in the gas phase and how tungsten transport processes might affect the supply of barium from the interior of the insert are not understood. This section describes experimental findings that guided the development of the barium transport model.

\section{A. Experimental approach}

The cathode inserts from the $8200 \mathrm{~h}$ LDT and the 30352 h ELT had previously been fractured along their axes to allow inspection. ${ }^{3,5}$ In the investigation summarized here the LDT discharge and neutralizer cathode inserts, the ELT discharge cathode insert, and a control insert that had not been operated were further analyzed to gain more insight into material transport and its effect on the emission zone. The profiles of the interior surfaces were measured using a laser profilometer system with a resolution of less than $1 \mu \mathrm{m}$. The profilometer calibration was checked by scanning a series of steps made with precision ground ceramic blocks of different heights that had been rung onto an optical flat.

The surface morphology and internal porosity of the inserts and the discharge cathode orifice plates were characterized in examinations with a scanning electron microscope (SEM). The composition of the impregnant and various deposits was determined using energy-dispersive spectroscopy (EDS). Several insert samples were potted to allow a fracture surface to be polished. This was done with very fine diamond abrasives wetted with kerosene to avoid removing impregnant material from the pores. SEM exams and EDS analysis were then used to characterize the cathode bulk. In particular, element maps of the interior were created with EDS scans to reveal the distribution of impregnant material.

\section{B. Erosion and deposition sites}

The profiles of the insert inner surfaces are shown in Fig. 1. These are based on profilometer scans along the axis of the insert fragments, which yielded the surface height above a flat reference plane on which the insert fragment rested. The height values were shifted so that zero height represents the original interior surface and the erosion depth (or increase in inner radius of the cylindrical insert) is indicated by negative values. The original surface datum was clear in the control 


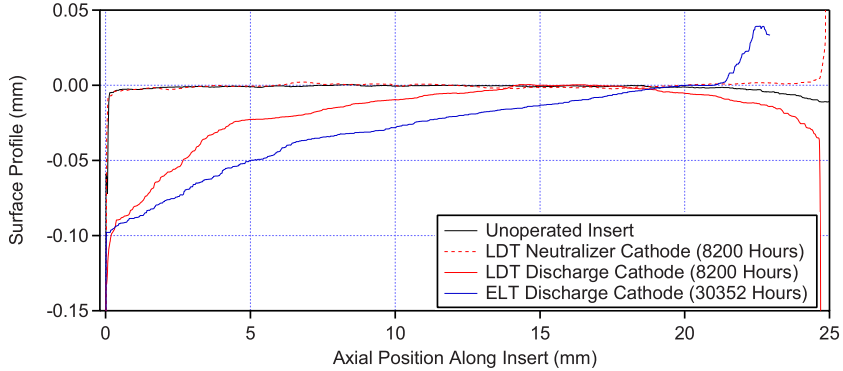

FIG. 1. (Color online) Axial profiles of four cathode inserts showing erosion of the interior surface.

insert and the neutralizer insert, which exhibited very little internal erosion. For the discharge cathode inserts, however, points near the upstream end where SEM exams showed surfaces that did not appear to be eroded or covered with deposits were assumed to be the original, uneroded surface. This is somewhat subjective, however, so the magnitude of the erosion depths indicated by negative values on the abscissa may be underestimated.

The control insert surface profile was flat to within $\pm 5 \mu \mathrm{m}$ except near the upstream end where the indicated height drops slightly, which represents an increase in the insert inner radius of about $15 \mu \mathrm{m}$. This feature is near the interface between the porous tungsten insert and a molybdenum ring that is brazed on the upstream end, and may be an artifact of machining. The LDT neutralizer insert profile is almost identical, except that it shows a smooth, filleted transition to the molybdenum ring. The LDT discharge cathode profile exhibits erosion over $15 \mathrm{~mm}$ at the downstream end, with more severe erosion in the first $4-5 \mathrm{~mm}$. Approximately $74 \mathrm{mg}$ of tungsten was eroded from this region, which is equivalent to $4 \times 10^{-4}$ moles with an average erosion rate of $1.5 \times 10^{-9} \mathrm{~g} / \mathrm{cm}^{2} \mathrm{~s}$. The radius of this insert is also larger near the upstream end. It is not clear if this is the same kind of feature seen in the control insert, or if it indicates another erosion site.

The ELT discharge cathode insert is qualitatively similar to the LDT insert at the downstream end, although the erosion is generally deeper and the transition in the slope of the eroded surface occurs at about $7 \mathrm{~mm}$ from the tip. The total erosion in the ELT insert was approximately $126 \mathrm{mg}$ or 6.9 $\times 10^{-4}$ moles with an average erosion rate of 5.2 $\times 10^{-10} \mathrm{~g} / \mathrm{cm}^{2} \mathrm{~s}$ over the course of the test. Both profiles indicate a maximum eroded depth at the tip of $100 \mu \mathrm{m}$, which yields a peak erosion rate of $1.4-5.2 \times 10^{-9} \mathrm{~g} / \mathrm{cm}^{2} \mathrm{~s}$. The ELT insert had thick deposits of barium compounds on the last $3 \mathrm{~mm}$ of the upstream end, which is seen in the profile as an increase in height of about 30-40 $\mu \mathrm{m}$ over the original surface.

Figure 2(a) is a composite photo constructed from SEM images of the LDT discharge cathode cross section showing the first $5 \mathrm{~mm}$ of the downstream end. The upper surface is the interior of the cylindrical insert and the white line indicates the original surface, highlighting the erosion in the emission zone.

The insert manufacturing process includes a weak etch after impregnation to remove impregnant from the surface

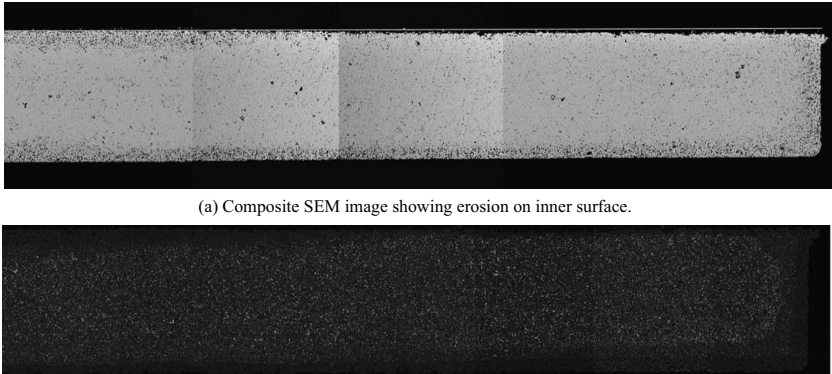

(b) Barium element map showing the distribution of barium in the interior

FIG. 2. Cross section of the cathode insert showing erosion and barium distribution at the downstream end.

and pores near the surface. This prevents excess barium production early in cathode operation. The surface layer from which the impregnant has been removed is apparent in the photo as a region with dark pores. The porosity of the tungsten matrix is actually very uniform throughout the insert, but in this region the pores are emphasized because they are filled with potting material, which looks dark in the secondary electron imaging mode. The impregnant-free layer has been removed from the emitting zone by erosion.

Figures 3-6 summarize SEM exams of the inner insert surface morphology. The morphology of the control insert does not vary over the inner surface. On this scale a large number of pores with a diameter of $1-5 \mu \mathrm{m}$ are visible, as shown in Fig. 3.

The morphology of the ELT discharge cathode insert varies considerably with position, as shown in Fig. 4. Photomicrographs A and B show tungsten crystals that were noted earlier ${ }^{5}$ and have been observed in many long duration tests. ${ }^{3,11,12}$ What is most striking about these photomicrographs is that they reveal a dramatic restructuring of the surface. This is evidently not only a site of erosion, but also of subsequent redeposition, resulting in a net recession of the surface accompanied by densification of the matrix. In this region the surface porosity is much lower than the original surface. Image $\mathrm{C}$ represents a transition from the region with crystalline deposits to the original porous structure. Image D

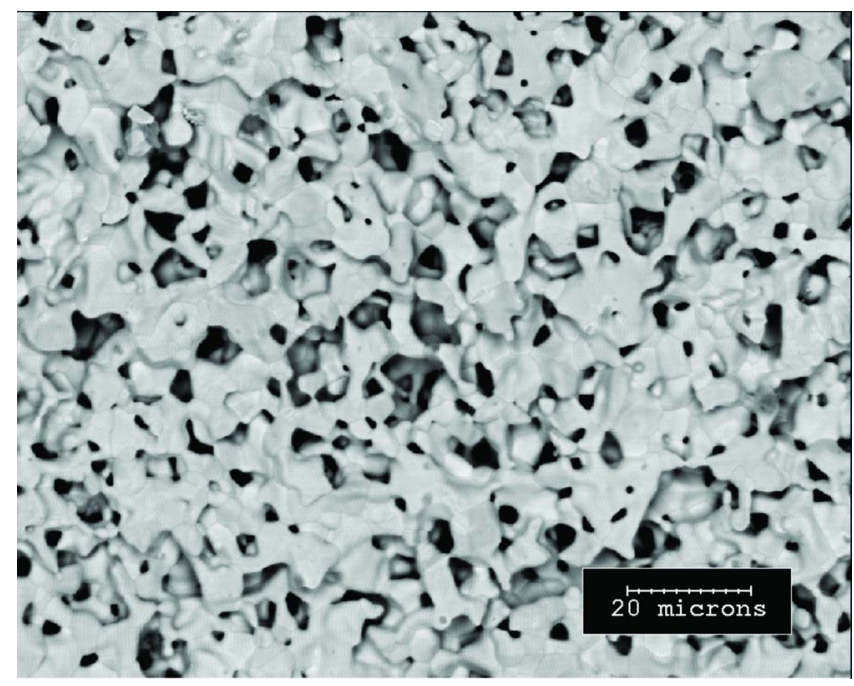

FIG. 3. (Color online) SEM image of the control insert inner surface 

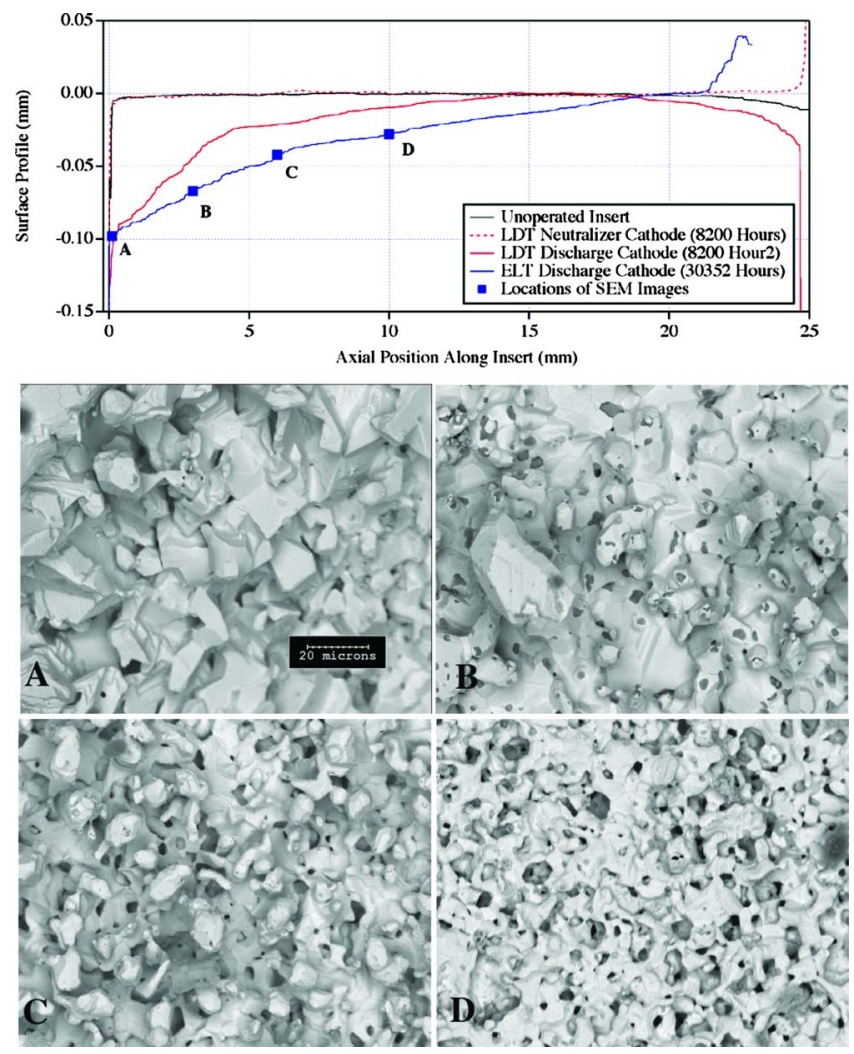

FIG. 4. (Color online) SEM images of the ELT discharge cathode insert inner surface.

shows that by $10 \mathrm{~mm}$ from the downstream end, the surface looks very similar to that of the unoperated insert, although the profile data indicate that net erosion occurred here. With the exception of the thick barium deposits found on the upstream end of the insert, very little barium was observed on the surface.

Six distinct zones found along the axis of the LDT insert are identified in Fig. 5. A region about $2 \mathrm{~mm}$ long at the downstream end of the insert tube was densely covered with fine tungsten crystals and a less dense covering of much larger crystals, as shown in the photomicrograph in Fig. 6. This image is at a lower magnification but is qualitatively

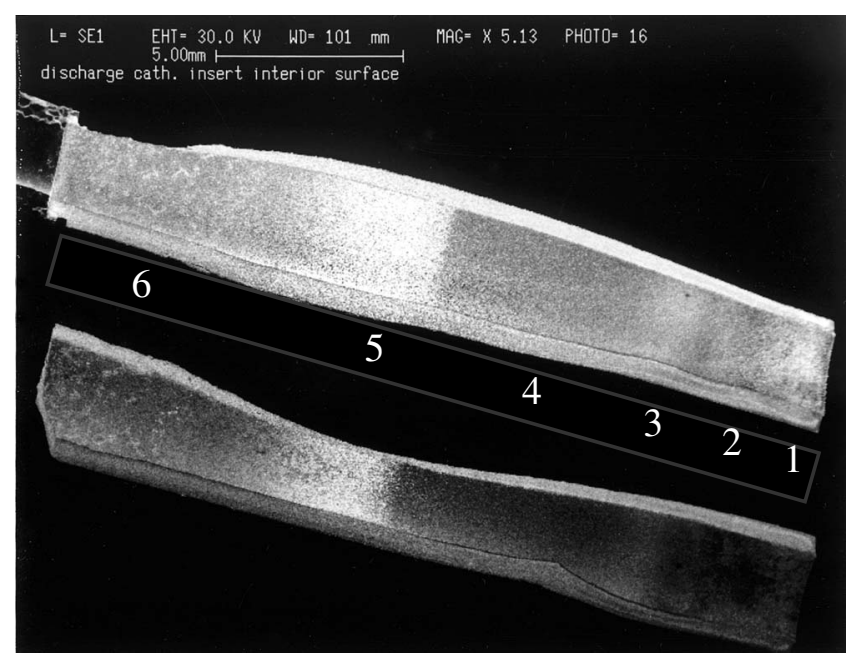

FIG. 5. Six zones on the interior of the discharge cathode insert.

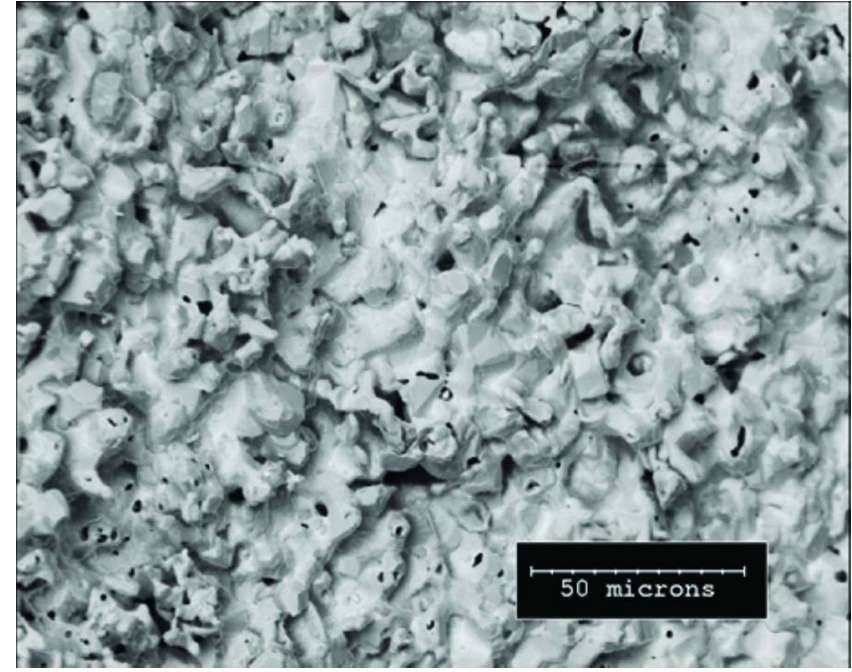

FIG. 6. (Color online) SEM image of the LDT discharge cathode insert inner surface near the downstream end.

similar to those from the ELT, showing the formation of a dense tungsten layer by some combination of erosion and redeposition at the downstream end. This coating also appeared to significantly reduce the porosity of the surface in this region. Some areas with barium deposits were found in this zone.

A $2 \mathrm{~mm}$ region upstream of the first zone was also covered with the small crystals and some large ones, but had very little barium on the surface. A third region about 1.7 $\mathrm{mm}$ long upstream of that had a surface similar to a new sintered insert with some of the smaller crystals. This region also had some barium deposits in the matrix. A region about $5.3 \mathrm{~mm}$ long in the center of the insert tube was composed of a sintered porous tungsten matrix with very little evidence of barium. The surface of a fifth region about $3.7 \mathrm{~mm}$ long near the upstream end of the insert was almost completely covered with a coating containing barium, calcium, aluminum, and oxygen (presumably the original impregnant material). The upstream end was primarily the tungsten matrix with isolated patches of barium, calcium, aluminum, and oxygen in the pores.

\section{Impact on barium transport}

A significant effort was made to determine the distribution of impregnant materials to help understand barium depletion and transport. Because the impregnant is found primarily in the pores, measurements of the distribution of the impregnant species $\mathrm{Ba}, \mathrm{Ca}, \mathrm{Al}$, and $\mathrm{O}$ at small scales tend to be dominated by local fluctuations between the pores and the tungsten matrix. EDS line scans along fracture or cross section surfaces are generally too noisy to extract meaningful information on the distribution of barium-containing materials beneath the surface. In this case we were successful in visualizing the distribution in the interior of the LDT discharge cathode with EDS element maps and in reconstructing element profiles with depth by integrating map data over relatively wide lateral swaths to average out fluctuations in signal intensity due to local nonuniformities in density. 


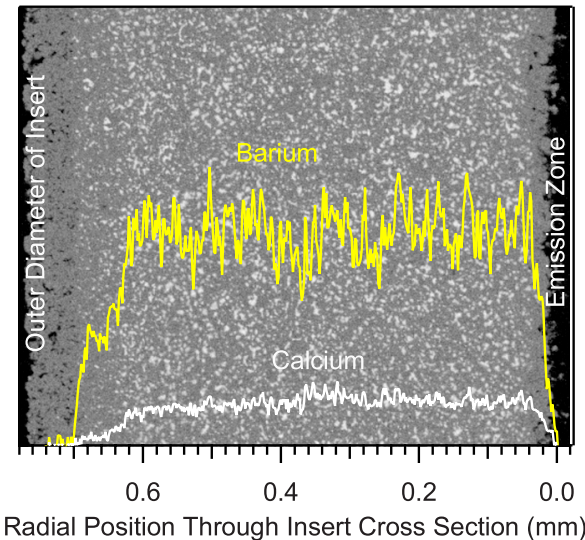

FIG. 7. (Color online) Barium element map and profiles for barium and calcium in a cross section of the LDT cathode.

Figure 2(b) shows the barium element map for the cross section pictured in Fig. 2(a). As expected, the image is dark in the potting material around the insert and in the impregnant-free surface layer noted in Fig. 2(a). The signal intensity is highest deep in the interior, where there is unreacted impregnant. The important feature in this image is the region with an intermediate signal strength. This zone is found in the upper left, under the impregnant-free layer, in the upper right corner, the downstream face on the right side and along the bottom between the impregnant-free layer, and the unreacted impregnant. However, this zone is not visible under the eroded zone in the upper right. Here the brightest zone extends nearly to the surface.

This is shown quantitatively in Fig. 7. The image is a barium element map of a region on the cross-sectioned LDT discharge cathode insert approximately $0.8 \mathrm{~mm}$ long located just upstream of the tip. The right side is the electron emission zone on the inner diameter of the insert and the left side is the outer diameter of the insert. The bright points are concentrations of barium in the tungsten matrix. The tungsten shows as a faint background in this image.

The barium-free zone where the insert was etched after impregnation is faintly visible on the left side, but has been mostly removed by erosion on the right. The yellow line is the sum of the columns composing the image and represents the average barium signal strength at each radial location. This analysis method eliminates much of the noise that would be associated with a line scan across the sample. The white trace is based on a similar analysis of the calcium element map. The tungsten background has been subtracted from these signals. On the left side of the image is an intermediate layer where the barium signal drops to about half that in the interior and the calcium signal drops nearly to zero. This is the zone from which impregnant material has been depleted by reactions with the tungsten. The space between the insert and the cathode tube was filled with a layer of $\mathrm{BaO}$, which is undoubtedly the result of reaction products flowing out from this reaction zone. On the right side of the image a thin region with little or no impregnant is visible, but there is no depletion layer as on the left. The barium and calcium signals are uniform up to the tungsten layer. Upstream of the region with the heaviest erosion the depletion layer can be found on both surfaces of the cathode, although the layer thickness decreases with increasing distance upstream.

The picture that emerges from these data is that material is eroded from the insert, particularly in the emission site at the downstream end, but some fraction of it is redeposited locally. The local redeposition in the emission zone forms a much denser tungsten shell that impedes the flow of barium from the interior, inhibiting the reduction in the impregnant under the emission zone. Barium lost from the emitter by evaporation in this zone must be replenished through the gas phase by barium released further upstream.

Further evidence for this was obtained in an experiment performed on Qinetiq T6 ion engine discharge cathodes. ${ }^{15}$ To simulate heavily depleted emitters, several special two-piece inserts were fabricated in which the upstream segment was a conventional impregnated porous tungsten tube and the downstream porous tungsten segment (either $25 \%$ or $50 \%$ of the total length) was not impregnated. Cathodes with these inserts ignited easily and appeared to operate just like cathodes with normal inserts. This strongly suggests that the downstream portion of the emitter can be supplied with barium through the gas phase.

\section{BARIUM TRANSPORT IN THE HOLLOW CATHODE INTERNAL PLASMA}

The purpose of this model is to understand barium transport in the xenon plasma and in particular how the cathode operates when a dense tungsten deposit on the downstream end prevents barium flow from the interior of the insert. The initial focus is on barium because this is the dominant species produced by the insert, ${ }^{16}$ although the model will be extended to include $\mathrm{BaO}$ flow and chemistry. Barium transport is strongly influenced by the xenon plasma; the electron density and temperature control the ionization of barium and the electric field in the xenon plasma and collisions with Xe neutrals and Xe ions control the diffusion of barium. However, barium atom and ion densities are so low that they do not significantly modify the charge density or temperature in what is primarily a xenon discharge. This allows us to separate the problem into two parts and use the major species parameters predicted by a xenon discharge model as fixed quantities in the solution of the minor species transport equations.

\section{A. Minor species transport equations}

The momentum equation for species $i$ can be written as

$$
\begin{aligned}
\frac{\partial\left(n_{i} m_{i} \mathbf{u}_{i}\right)}{\partial t}+\nabla \cdot\left(n_{i} m_{i} \mathbf{u}_{i} \mathbf{u}_{i}\right)= & -\nabla P_{i}-\nabla \cdot \tau_{i}+m_{i}\left(S_{i} \mathbf{u}_{i \text {,irth }}\right. \\
& \left.-R_{i} \mathbf{u}_{i, \text { death }}\right)+n_{i} q_{i}\left(\mathbf{E}+\mathbf{u}_{i}\right. \\
& \times \mathbf{B})+\sum_{j} n_{i} \nu_{i j} \mu_{i j}\left(\mathbf{u}_{i}-\mathbf{u}_{j}\right),
\end{aligned}
$$

where $n_{i}$ is the density of species $i, m_{i}$ is the mass, $\mathbf{u}_{i}$ is the velocity, $P_{i}$ is the partial pressure, $\tau_{i}$ is the viscous stress tensor, $S_{i}$ is the rate at which species $i$ particles are created 
(by ionization reactions, for instance), $\mathbf{u}_{i \text {,birth }}$ is the average velocity of newly created $i$ particles, $R_{i}$ is the rate at which $i$ particles are lost (through recombination, for example) and $\mathbf{u}_{i \text {,death }}$ is the mean velocity when they are lost, $q_{i}$ is the particle charge, and $\mathbf{E}$ is the electric field and $\mathbf{B}$ is the magnetic field. The final term represents momentum lost from species $i$ in collisions with species $j$, which is proportional to the collision frequency $\nu_{i j}$, the reduced mass $\mu_{i j}$, and the mean relative velocity between the populations. The species continuity equation is

$$
m_{i} \frac{\partial n_{i}}{\partial t}+m_{i} \nabla \cdot\left(n_{i} \mathbf{u}_{i}\right)=m_{i}\left(S_{i}-R_{i}\right)=m_{i} \dot{n}_{i}
$$

where $\dot{n}_{i}$ is the net rate at which species $i$ is created.

Expanding the derivatives in the momentum equation reveals terms identical to the left-hand side of the continuity equation multiplied by the species velocity. The terms on the right-hand side of the momentum equation that are associated with particle creation and loss are identical to the righthand side of the continuity equation multiplied by the mean species velocity if we assume $\mathbf{u}_{i \text {,birth }}=\mathbf{u}_{i \text {,death }}=\mathbf{u}_{i}$. These terms can therefore be subtracted out. If we neglect the viscous term and magnetic field effects, the momentum equation simplifies to

$n_{i} m_{i} \frac{\partial \mathbf{u}_{i}}{\partial t}+n_{i} m_{i} \mathbf{u}_{i} \cdot \nabla \mathbf{u}_{i}=-\nabla P_{i}+n_{i} q_{i} \mathbf{E}+\sum_{j} n_{i} \nu_{i j} \mu_{i j}\left(\mathbf{u}_{j}-\mathbf{u}_{i}\right)$

If the phenomena of interest are sufficiently slow, i.e., there are no high frequency phenomena and fluid acceleration is small compared to the force terms, we may neglect the momentum terms on the left-hand side and the resulting equation can be solved for the species flux, $\boldsymbol{\Gamma}_{i}=n_{i} \mathbf{u}_{i}$. For example, if we write Eq. (3) for barium ions $\left(\mathrm{Ba}^{+}\right)$including momentum transfer collisions with electrons $(e)$, xenon ions $\left(\mathrm{Xe}^{+}\right)$ and xenon neutrals $(\mathrm{Xe})$, we obtain

$$
\begin{aligned}
0= & -\nabla P_{\mathrm{Ba}^{+}}+e n_{\mathrm{Ba}^{+}} \mathbf{E}-n_{\mathrm{Ba}^{+}}\left[\nu_{\mathrm{Ba}^{+} e} \mu_{e \mathrm{Ba}}\left(\mathbf{u}_{\mathrm{Ba}^{+}}-\mathbf{u}_{e}\right)\right. \\
& \left.+\nu_{\mathrm{Ba}^{+} \mathrm{Xe}^{+}} \mu_{\mathrm{BaXe}}\left(\mathbf{u}_{\mathrm{Ba}^{+}}-\mathbf{u}_{\mathrm{Xe}^{+}}\right)+\nu_{\mathrm{Ba}^{+} \mathrm{Xe}} \mu_{\mathrm{BaXe}}\left(\mathbf{u}_{\mathrm{Ba}^{+}}-\mathbf{u}_{\mathrm{Xe}}\right)\right] .
\end{aligned}
$$

Collisions between barium ions and other minor species (such as $\mathrm{Ba}$ and $\mathrm{BaO}$ ) are assumed to be sufficiently rare to neglect. Recognizing that $\mathbf{u}_{e} \gg \mathbf{u}_{\mathrm{Ba}^{+}}$, that $\mathbf{u}_{\mathrm{Xe}}$ is negligible, and that $\mu_{e \mathrm{Ba}} \simeq m_{e}$, we obtain

$$
\begin{aligned}
0= & -\nabla P_{\mathrm{Ba}^{+}}+e n_{\mathrm{Ba}^{+}} \mathbf{E}-n_{\mathrm{Ba}^{+}}\left[-\nu_{\mathrm{Ba}^{+} e} m_{e} \mathbf{u}_{e}\right. \\
& +\nu_{\mathrm{Ba}^{+} \mathrm{Xe}^{+}} \mu_{\mathrm{BaXe}}\left(\mathbf{u}_{\mathrm{Ba}^{+}}-\mathbf{u}_{\mathrm{Xe}^{+}}\right)+\nu_{\mathrm{Ba}^{+} \mathrm{Xe}} \mu_{\left.\mathrm{BaXe} \mathbf{u}_{\mathrm{Ba}^{+}}\right] .}
\end{aligned}
$$

Solving this equation for the barium ion flux yields

$$
\begin{aligned}
& \boldsymbol{\Gamma}_{\mathrm{Ba}^{+}}=n_{\mathrm{Ba}^{+}} \mathbf{u}_{\mathrm{Ba}^{+}}=\frac{-\nabla P_{\mathrm{Ba}^{+}}+e n_{\mathrm{Ba}^{+}} \mathbf{E}}{\nu_{\mathrm{Ba}^{+} \mathrm{Xe}^{+}} \mu_{\mathrm{BaXe}}\left(1+\nu_{\mathrm{Ba}^{+} \mathrm{Xe}} / \nu_{\mathrm{Ba}^{+} \mathrm{Xe}^{+}}\right)}
\end{aligned}
$$

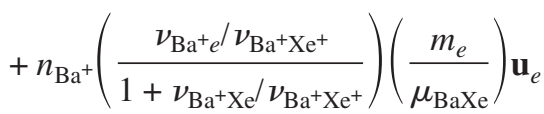

$$
\begin{aligned}
& +n_{\mathrm{Ba}^{+}}\left(\frac{1}{1+\nu_{\mathrm{Ba}^{+} \mathrm{Xe} / \nu_{\mathrm{Ba}^{+} \mathrm{Xe}^{+}}}}\right) \mathbf{u}_{\mathrm{Xe}^{+}} \text {. }
\end{aligned}
$$

The first term represents ion flux driven by pressure gradient and electrostatic forces. The second and third terms are the fluxes driven by drag due to collisions with electrons and xenon ions, respectively. The collision frequency terms are cast in the form of ratios to the dominant interaction, which is coulomb collisions between xenon and barium ions. The electron drag term can be neglected because of the small electron-to-heavy particle mass ratio.

The same approach yields the flux of neutral barium atoms,

$$
\begin{aligned}
\boldsymbol{\Gamma}_{\mathrm{Ba}}= & n_{\mathrm{Ba}} \mathbf{u}_{\mathrm{Ba}}=\frac{-\nabla P_{\mathrm{Ba}}}{\nu_{\mathrm{BaXe}} \mu_{\mathrm{BaXe}}\left(1+\nu_{\mathrm{BaXe}} / \nu_{\mathrm{BaXe}}\right)} \\
& +n_{\mathrm{Ba}}\left(\frac{\nu_{\mathrm{BaXe}^{+}} / \nu_{\mathrm{BaXe}}}{1+\nu_{\mathrm{BaXe}^{+}} / \nu_{\mathrm{BaXe}}}\right) \mathbf{u}_{\mathrm{Xe}^{+} .}
\end{aligned}
$$

Electron drag has also been neglected in this equation.

The ion-ion collision frequency that appears in these equations is given by the standard coulomb frequency,

$$
\begin{aligned}
\nu_{\mathrm{Ba}^{+} \mathrm{Xe}^{+}}= & \frac{\sqrt{2}}{3 \sqrt{\pi}} n_{\mathrm{Xe}^{+}} \\
& \times\left(\frac{e^{2}}{4 \pi \epsilon_{0}}\right)^{2} \frac{4 \pi}{m_{\mathrm{Ba}}^{1 / 2}\left(k T_{h}\right)^{3 / 2}}\left(\frac{m_{\mathrm{Xe}}}{m_{\mathrm{Ba}}+m_{\mathrm{Xe}}}\right)^{1 / 2} \ln \Lambda_{i} .
\end{aligned}
$$

The neutral barium-xenon collision frequency is expressed in terms of a constant scattering cross section $\sigma_{D}=40 \AA^{2}$ based on diffusion measurements for barium in xenon. ${ }^{17}$

$$
\nu_{\mathrm{BaXe}}=n_{\mathrm{Xe}} \sigma_{D}\left(\frac{8 k T_{h}}{\pi \mu_{\mathrm{BaXe}}}\right)^{1 / 2} .
$$

$\mathrm{Ba}$ ion-Xe neutral and $\mathrm{Ba}$ neutral-Xe ion collisions are assumed to have the same cross section as the neutral-neutral collisions.

The continuity equations for barium ions and neutrals can be expressed in terms of the fluxes,

$$
\begin{aligned}
& \frac{\partial n_{\mathrm{Ba}^{+}}}{\partial t}+\nabla \cdot \boldsymbol{\Gamma}_{\mathrm{Ba}^{+}}=\dot{n}_{\mathrm{Ba}^{+}}, \\
& \frac{\partial n_{\mathrm{Ba}}}{\partial t}+\nabla \cdot \boldsymbol{\Gamma}_{\mathrm{Ba}}=\dot{n}_{\mathrm{Ba}}=-\dot{n}_{\mathrm{Ba}^{+}},
\end{aligned}
$$

where $\dot{n}_{\mathrm{Ba}^{+}}$is the rate at which barium ions are created by ionization of barium neutrals and $\dot{n}_{\mathrm{Ba}}$ is the rate at which neutrals are consumed by ionization reactions. The ionization rate is given by the product of the barium atom density and the ionization rate coefficient, $\dot{n}_{\mathrm{Ba}^{+}}\left(T_{e}\right)=n_{\mathrm{Ba}} \nu_{\mathrm{Ba}}^{i z}$. The ionization rate coefficient for $\mathrm{Ba}$ was obtained by integrating mea- 


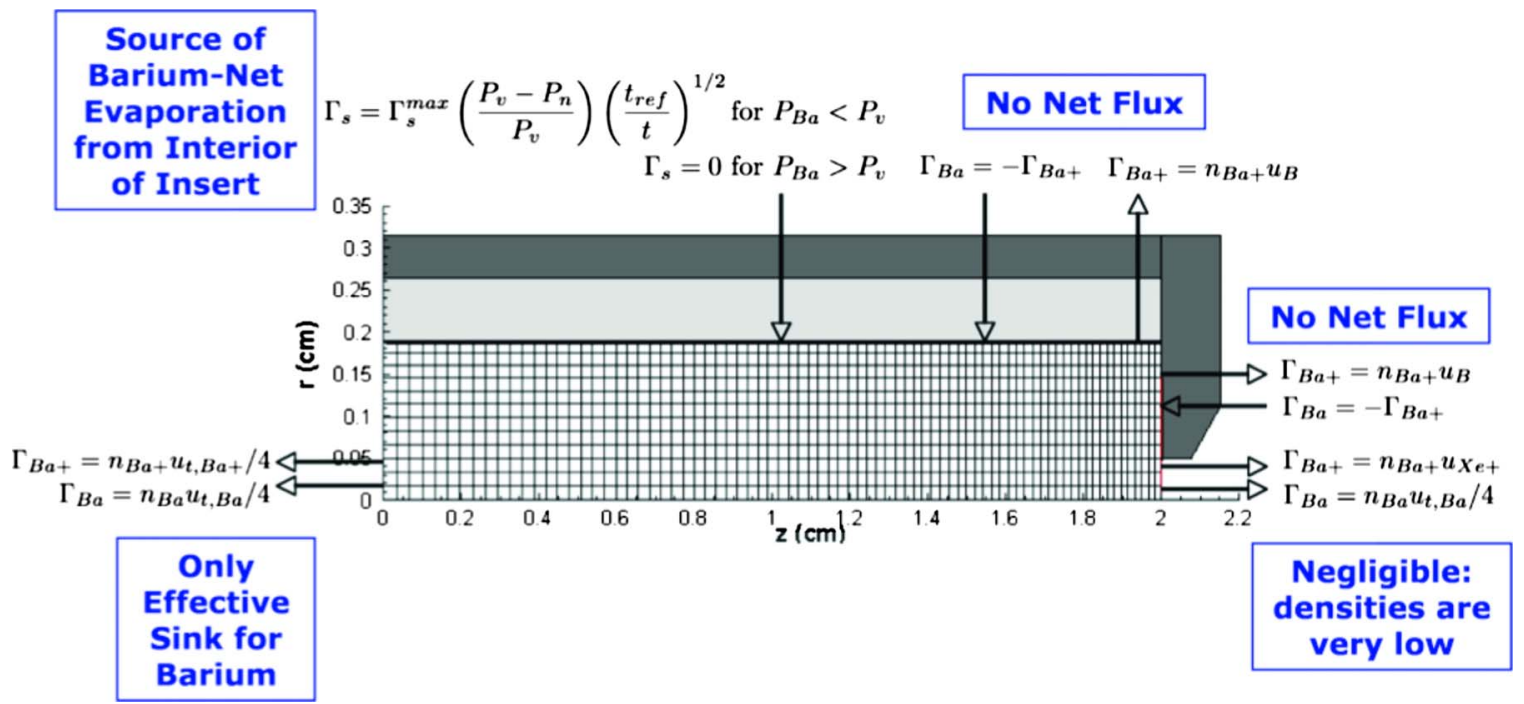

FIG. 8. (Color online) Boundary conditions and computational grid for the barium transport model.

sured barium ionization cross sections ${ }^{18}$ over a Maxwellian velocity distribution,

$$
\nu_{\mathrm{Ba}}^{i z}=n_{e}\left\langle\sigma_{i} \mathbf{u}_{e}\right\rangle .
$$

The model also employs the ideal gas equation of state. The barium ions and neutrals are assumed to equilibrate with the xenon atoms and ions, so all heavy particles have a single temperature $T_{h}$.

\section{B. Boundary conditions}

The continuity equations are solved on a twodimensional axisymmetric region $2 \mathrm{~cm}$ long encompassing the insert plasma at the downstream end of the emitter. The simulation domain is bounded by five surfaces as shown in Fig. 8, including the centerline, the entrance to the cathode orifice, the upstream face of the orifice plate, the emitter surface, and the upstream inlet to the cathode. Ion and neutral fluxes through these surfaces are specified as boundary conditions.

Centerline. The centerline is a symmetry boundary, so the ion and neutral fluxes are set equal to zero.

Emitter surface. The flow rate through the emitter surface is equal to the rate at which barium produced in the interior is transported through the cathode matrix in steady state. Barium is generated by reactions between the impregnant and tungsten on the pore walls. The source is assumed to be a reaction front which propagates into the cathode interior over time. Below the reaction front is unreacted impregnant material; above the front the pores of the tungsten matrix are filled with a porous material consisting of the stable solid phase reaction products. The gaseous barium products generated at the reaction front flow through this porous structure by Knudsen flow or surface diffusion. The flow is driven by the difference in the pressure at the reaction front and the barium partial pressure at the insert surface. Diffusion through the matrix is assumed to be rate controlling, resulting in a strongly diffusion-limited impregnant decomposition reaction at the front. The barium partial pressure at the reaction front will therefore be approximately equal to the equilibrium pressure of barium over the reactants, which is a function of the insert temperature. As barium in the impregnant is consumed and the reaction front propagates into the interior, the gaseous barium must flow an everincreasing distance through the porous structure. The flow resistance through the pores will therefore increase with time. The rate at which barium is dispensed therefore depends on three main parameters: the insert temperature $T_{c}$, which controls the equilibrium barium pressure $P_{v}$ at the reaction front, the barium partial pressure in the insert plasma $P_{\mathrm{Ba}}$, and time $t$.

This reaction front model is an approximation of the true situation, in which the reaction probably occurs over some finite length of the diffusion path, but reproduces the observed time dependence of barium production. In vacuum cathodes, the barium supply rate is found to decrease as $t^{-1 / 2}$, which is consistent with Knudsen flow through a flow resistance which increases as barium is lost and the reaction front recedes into the surface. ${ }^{19,20}$

Barium transport through the cathode matrix is not modeled explicitly. Instead, we use a phenomenological description which relates the barium flow at time $t$ to measured supply rates at a reference time $t_{\text {ref }}$. Knudsen flow through the pores is proportional to the pressure difference,

$$
\Gamma_{e}\left(T_{c}, P_{\mathrm{Ba}}, t\right)=\Gamma_{e}\left(T_{c}, P_{\mathrm{Ba}}=0, t=t_{\mathrm{ref}}\right)\left(\frac{P_{v}-P_{\mathrm{Ba}}}{P_{v}}\right)\left(\frac{t_{\mathrm{ref}}}{t}\right)^{1 / 2} .
$$

The reference flux $\Gamma_{e}\left(T_{c}, P_{\mathrm{Ba}}=0, t=t_{\mathrm{ref}}\right)$ in atoms $/ \mathrm{cm}^{2} \mathrm{~s}$ is based on measurements ${ }^{2}$ of the barium supply rate from $S$-type cathodes in vacuum early in life (with a total operating time $t_{\text {ref }}$ on the order of $10 \mathrm{~h}$ ) as a function of cathode temperature $T_{c}$ in $K$,

$$
\ln \Gamma_{s}\left(T_{c}, P_{\mathrm{Ba}}=0, t_{\mathrm{ref}}\right)=63.53-46237 / T_{c} .
$$

Xenon will diffuse into the porous tungsten in a gas discharge, but the mean free path for collisions between barium and xenon atoms is approximately one order of magnitude larger than the pore size. The barium transport will still be 


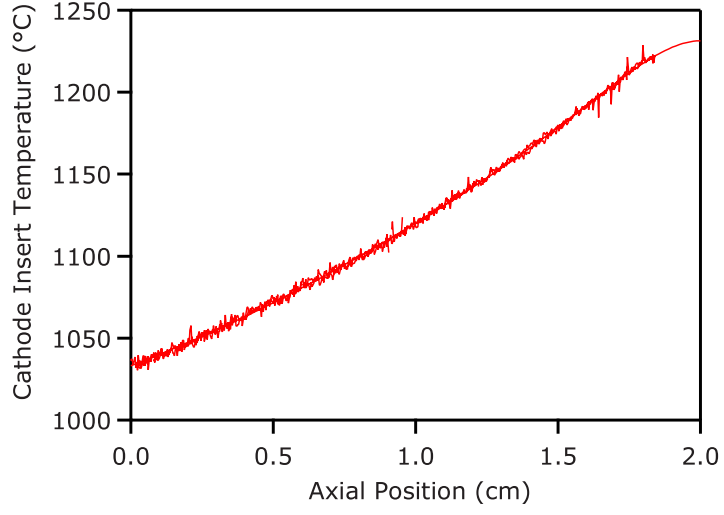

FIG. 9. (Color online) Axial temperature distribution along the insert inner surface.

dominated by interactions with the pore walls and the supply rate will therefore be similar to that measured under vacuum conditions. The temperature distribution measured along the axis of an emitter at the NSTAR full power operating condition shown in Fig. 9 is used in all of the simulations to calculate the barium flow rate as a function of position.

The equilibrium pressure $P_{v}$ in Torr for the impregnant decomposition reaction proposed by Rittner et al. ${ }^{19}$ is

$$
\log P_{v}=8.56-20360 / T_{c}
$$

The barium partial pressure at the surface $P_{\mathrm{Ba}}$ is determined by the solution to the barium diffusion equation given above. In cases for which the ambient barium pressure exceeds the equilibrium pressure, the reaction is assumed to cease.

Ba neutrals and ions also flow back to the insert surface. However, the cathode temperatures are too high for barium to accumulate in thick layers on the surface (the evaporation rate for bulk barium at these temperatures exceeds the ion and neutral fluxes by many orders of magnitude), so we assume that the surface has a steady state, submonolayer coverage of barium adsorbed on oxygen on tungsten. In steady state, there is no net barium flux to the surface. The neutral flux to the surface is balanced by the desorption of neutrals, and ions that strike the surface return to the plasma as neutral barium atoms. It is therefore a net neutral flow (in addition to the neutral flow from insert interior), which is equal in magnitude to the ion flow. This assumption is consistent with the observations from the ELT that barium does not accumulate on the downstream surfaces of the inserts.

Orifice plate upstream face. This surface was also assumed to have a steady-state, submonolayer coverage with a net neutral flow from the surface equal to the impinging ion flux.

Entrance to cathode orifice. Neutrals were assumed to flow out of the cathode orifice with their thermal velocity. Ions were assumed to have the same velocity at this point as the xenon ions. In practice, there are very few barium ions or neutrals in this region, so the results are not sensitive to these assumptions.

Upstream boundary. The upstream boundary is the only major sink for barium. Ions and neutrals were assumed to flow out this surface with their thermal velocities and con- dense on cooler surfaces upstream, as observed experimentally.,

\section{The background xenon plasma solution}

The barium transport model depends on a number of xenon plasma parameters, including the xenon ion and atom densities and heavy particle temperature, which appear in the collision frequencies, the electron density, and temperature, which determine the barium ionization rate, and the xenon ion velocity and electric field, which appear in the force terms of the momentum equations. These quantities are taken from a separate simulation of the xenon plasma and are assumed to be independent of the dynamics of the barium plasma.

The internal xenon plasma and neutral gas are modeled with eight governing equations that represent species continuity, momentum, and energy conservation. The equations are described in detail elsewhere ${ }^{21}$ and will only be summarized here. Continuity and momentum equations similar to Eqs. (2) and (3) are solved for xenon ions, xenon atoms, and electrons. The inertia terms are neglected in both the electron and ion momentum equations. The neutral atom bulk velocity is assumed to be negligible compared to the ion and electron bulk velocities, so the neutral gas dynamics are neglected. The functional forms of the collision frequencies and ionization rates are similar to those described above. Although previous work suggested that the classical resistivity may be enhanced anomalously by streaming instabilities downstream of the orifice entrance, ${ }^{22}$ only classical resistivity has been used in the present simulations. The comparisons with the measurements in this cathode suggest that classical resistivity is sufficient to characterize the plasma inside the emitter region. The system includes an electron energy equation that includes energy input from the electric field, convection, conduction, and energy transfer to the heavy species. It is assumed that ions and neutrals are in thermal equilibrium, so a single energy conservation equation which includes convection, conduction, and heat transfer from electrons is derived for the heavy species.

These equations yield the steady-state profiles of the following variables: plasma particle density $n_{\mathrm{Xe}^{+}}=n_{e}$, ion and electron current densities $\mathbf{j}_{i}$ and $\mathbf{j}_{e}$, respectively, electron temperature $T_{e}$, electric field $\mathbf{E}$, plasma potential $\phi$, neutral particle density $n_{\mathrm{Xe}}$, and heavy species temperature $T_{h}$. The total pressure $P$ is assumed to be uniform throughout the cathode channel and the neutral particle density is determined using the ideal gas law.

A mixture of flux and Dirichlet boundary conditions is used, depending on the quantity and the boundary. The electron flux from the insert boundary is given by thermionic emission at a specified cathode temperature according to the Richardson equation with a reduction in the work function due to the Schottky effect and an effective increase in emitting area due to surface morphology. ${ }^{23}$ Electrons with sufficient energy are allowed to flow from the plasma into conducting walls. Ions are assumed to flow into conducting surfaces with a flux $\Gamma_{\mathrm{Xe+}}=0.6 n_{\mathrm{Xe}^{+}} u_{B}$, where $u_{B}=\sqrt{k T_{e} / m_{\mathrm{Xe}}}$ is the Bohm velocity and the factor 0.6 accounts for accelera- 
tion of ions in the presheath. Ions are assumed to flow out of the upstream boundary at their thermal velocity. Gas temperatures are set equal to the walls and adiabatic conditions are enforced at the cathode inlet and orifice entrance. Electron heat conduction into material surfaces is set equal to zero while electrons absorbed from the plasma convect thermal energy and potential energy associated with the work function. The plasma density and potential on the centerline at the orifice entrance are specified and the potential of all conducting surfaces is set equal to zero (ground).

The conservation equations are discretized using finite volumes and solved on the computational mesh shown in Fig. 8. The flux vectors are edge centered and the scalar variables are cell centered. The fluxes are determined using second-order accurate finite differences. The system of equations is solved in a time-split manner using explicit time marching for the plasma particle density and electron temperature. Initial estimates of the electron current density vector field, plasma particle density, and electron temperature are used to compute all required fluxes, transport coefficients, and related quantities. The ion continuity and electron energy equations are then time marched to yield new values of $n_{e}$ and $T_{e}$. The evolution of these equations at fixed current density is repeated for $N$ iterations. When $N$ reaches a specified value, conservation of total current is solved implicitly to determine a new value of the plasma potential, which is in turn used to compute the new electric field $\mathbf{E}$. The electron current density vector field is then updated using the electron momentum equation. Concurrently, the heavy species energy equation is solved implicitly to determine the new heavy species temperature $T_{e}$. The procedure is repeated until the solution for all quantities has reached steady state. The five main inputs to the numerical model are the total discharge current, the internal cathode pressure $P$, the plasma density at the orifice boundary, the plasma potential at the orifice boundary, and the emitter temperature as a function of position. In the simulations the orifice plasma density has been specified based on measurements ${ }^{21}$ and the plasma potential at the orifice boundary is varied by iteration until the required discharge current is obtained.

The results of a simulation of the NSTAR full power point at a discharge current of $13.3 \mathrm{~A}$ and a xenon flow rate of 3.7 SCCM (SCCM denotes standard cubic centimeter at STP) are plotted in Fig. 10. Electron emission is concentrated at the downstream end of the emitter, producing a strong peak in plasma density. The high current density in the orifice results in a peak in the electron temperature on the centerline at the orifice entrance. The plasma potential is also peaked at the orifice and drops radially and axially. The heavy particle temperature peaks where the plasma density and electron temperature are highest. These distributions were used for all barium transport simulations reported in the next section.

The mean free path for barium atom ionization $\lambda_{\mathrm{mfp}}^{i z}$ $=u_{t, \mathrm{Ba}} / \nu_{\mathrm{Ba}}^{i z}$ based on the barium atom thermal velocity $u_{t, \mathrm{Ba}}$ $=\left(8 k T_{h} / \pi m_{\mathrm{Ba}}\right)^{1 / 2}$ and the ionization frequency was calculated using the electron density and temperature profiles and is plotted in Fig. 11. The mean free path for ionization upstream of the dense plasma region is of the order of meters,

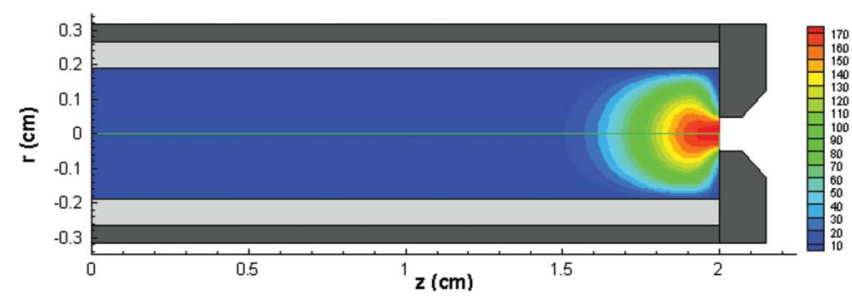

(a) Electron Density, $\mathrm{n}_{\mathrm{e}} / 10^{19}\left(\mathrm{~m}^{-3}\right)$

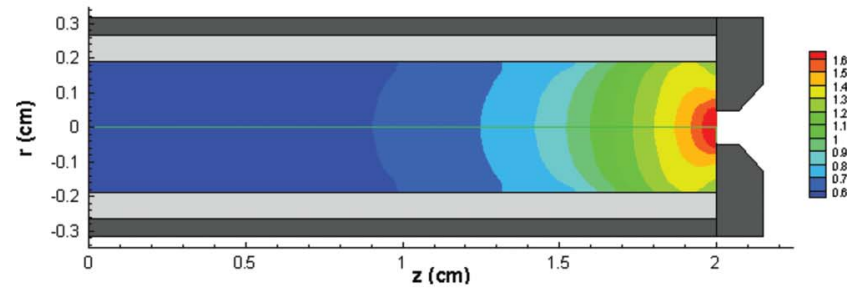

(b) Electron Temperature, $\mathrm{T}_{\mathrm{e}}(\mathrm{eV})$

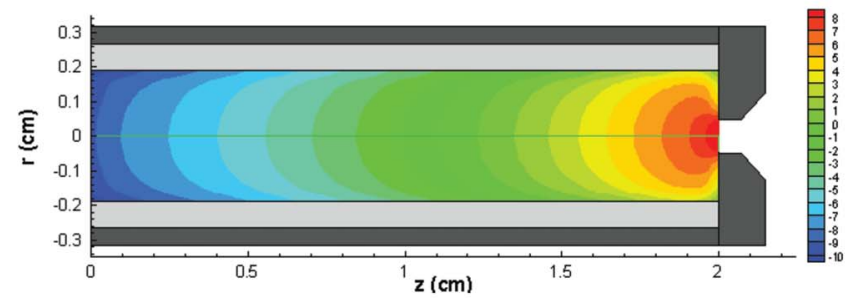

(c) Plasma Potential, $\phi(\mathrm{V})$

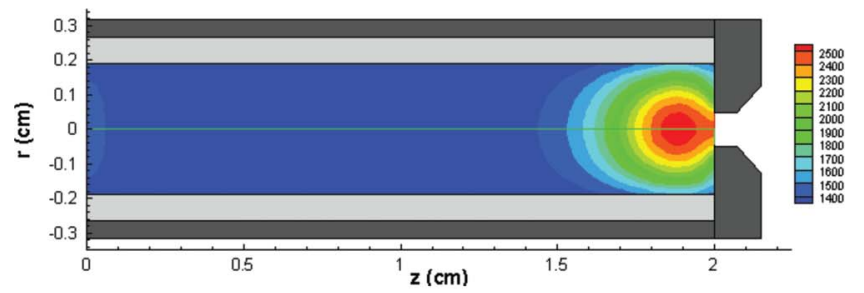

(d) Heavy Particle Temperature, $\mathrm{T}_{\mathrm{h}}(\mathrm{K})$

FIG. 10. (Color online) Distributions of key plasma parameters in the hollow cathode interior based on the xenon plasma model.

but drops to $0.1 \mathrm{~mm}$ near the orifice. Barium atoms that drift into the dense xenon plasma have a high probability of being ionized.

\section{Barium transport solutions}

The xenon plasma code was modified to solve a discretized form of Eqs. (10) and (11) on the same computational domain and mesh shown in Fig. 8. The barium ion and neutral densities were initially assumed to be a uniform low

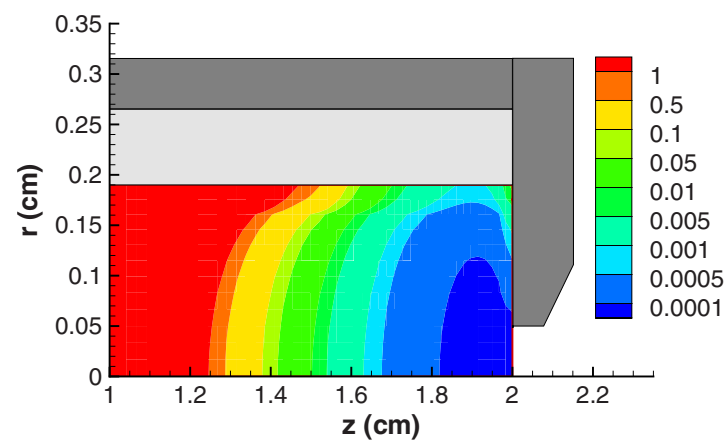

FIG. 11. (Color online) Mean free path for barium ionization $\lambda_{\mathrm{mfp}}^{i z}(m)$. 


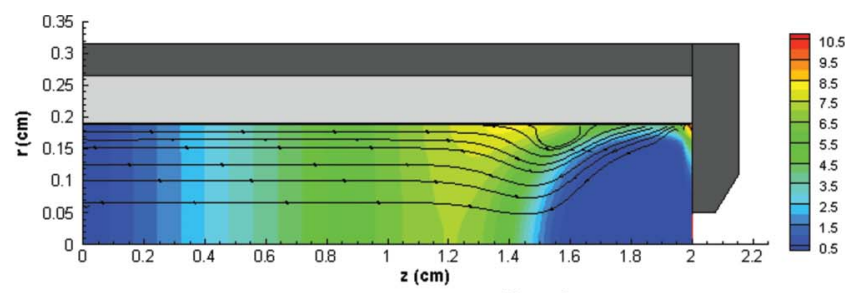

(a) Barium neutral density, $\mathrm{n}_{\mathrm{Ba}} / 10^{16}\left(\mathrm{~m}^{-3}\right)$, for Case 1

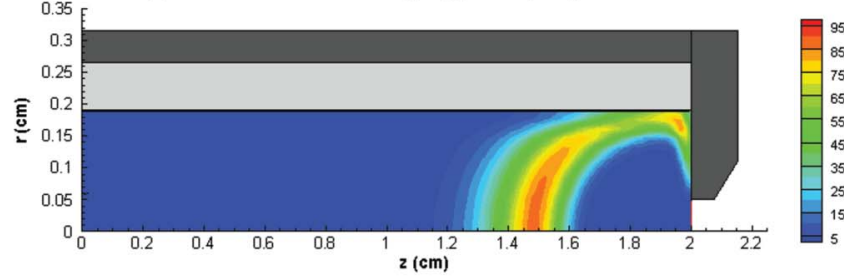

(b) Barium ion density, $\mathrm{n}_{\mathrm{Ba}^{\prime}} / 10^{14}\left(\mathrm{~m}^{-3}\right)$, for Case 1

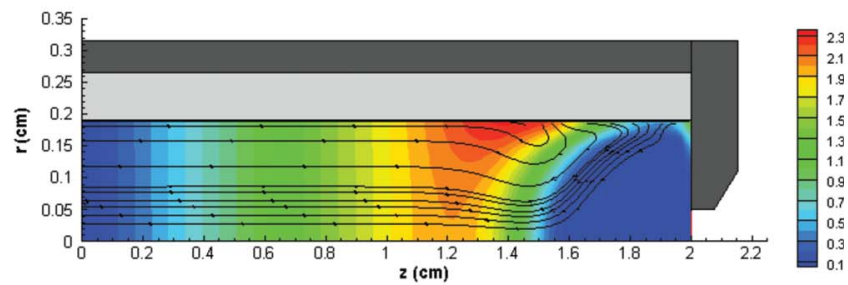

(c) Barium neutral density, $\mathrm{n}_{\mathrm{Ba}} / 10^{16}\left(\mathrm{~m}^{-3}\right)$, for Case 2

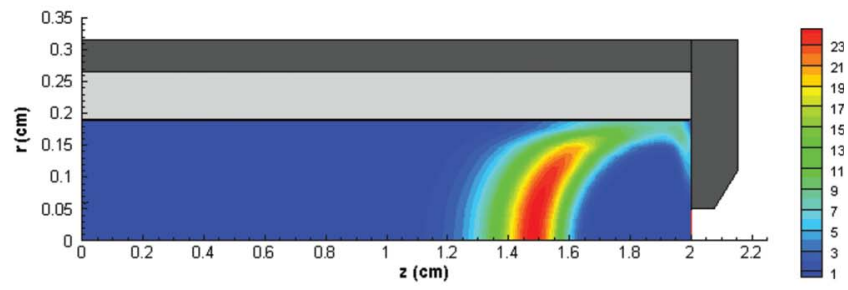

(d) Barium ion density, $\mathrm{n}_{\mathrm{Ba}} / 10^{14}\left(\mathrm{~m}^{-3}\right)$, for Case 2

FIG. 12. (Color online) Barium neutral and ion density distributions Streamlines on neutral density plots represent total barium flux (the sum of ion and neutral fluxes).

value and the continuity equations were then time marched until the densities and fluxes achieved steady state values. Two cases were simulated. In the first case, the entire insert surface was allowed to emit barium, except where the neutral barium pressure exceeded the equilibrium vapor pressure for the reaction. In the second case, the barium supply along the last $4 \mathrm{~mm}$ at the downstream end of the insert was set to zero to simulate the effect of pore closure by tungsten deposits. In both cases the accumulated operating time was assumed to be $1000 \mathrm{~h}$, so the barium supply rate was a factor of 10 times lower than that at the reference time of $10 \mathrm{~h}$ due to recession of the reaction front.

The neutral and ion density distributions for the two cases are plotted in Fig. 12. In both cases the distributions are qualitatively similar. Figures $12(\mathrm{a})$ and 12(c) show that the neutral density peaks near the emitter surface at about 1.4 $\mathrm{cm}$. This occurs because the emitter is the main source of barium neutrals. However, the neutral density drops to a very low value in a hemispherical region centered on the cathode orifice. Comparison with Fig. 11 reveals that this corresponds to the location in the discharge where the barium ionization mean free path is small. Barium neutrals emitted by the insert are consumed in ionization collisions when they

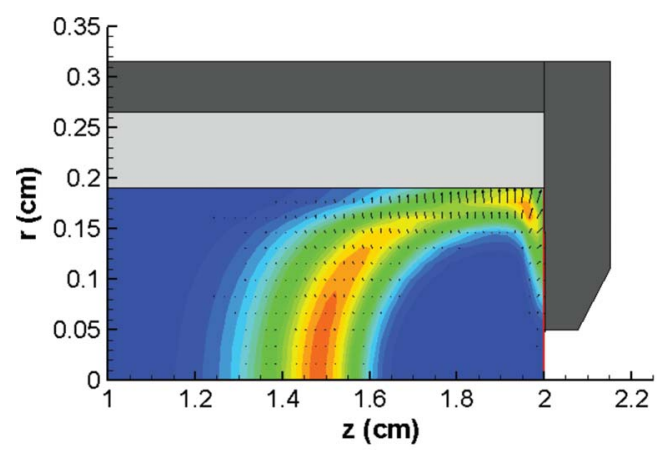

(a) Ion density, $\mathrm{n}_{\mathrm{Ba}^{+}} / 10^{14}\left(\mathrm{~m}^{-3}\right)$, and ion flux vectors, Case 1

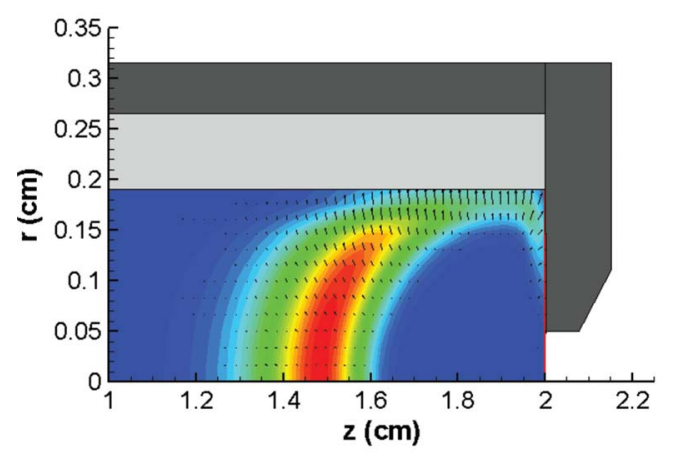

(b) Ion density, $\mathrm{n}_{\mathrm{Ba}^{+}} / 10^{14}\left(\mathrm{~m}^{-3}\right)$, and ion flux vectors, Case 2

FIG. 13. (Color online) Barium ion density distributions and ion flux vectors.

enter the intense xenon plasma and Figs. 12(b) and 12(d) demonstrate that the resulting ion distribution peaks in a thin ionization front at the periphery of the xenon discharge zone.

The streamlines plotted in Figs. 12(a) and 12(c) represent the total flow of barium, i.e., the sum of the barium ion and neutral fluxes, in the insert plasma. As expected, neutrals supplied by the insert or generated by neutralization of ions striking the conducting boundaries ultimately flow to the sink on the upstream boundary. The diffusive flux upstream of the $1 \mathrm{~cm}$ location is driven primarily by the density gradient. As the barium flow from the insert increases, the peak neutral density rises to produce a density gradient and efflux sufficient to balance the influx. The dynamics at the downstream end are more complex.

Figure 13 shows the ion density distributions with vectors that represent the ion fluxes. The ion fluxes are primarily in the positive $r$-direction, reflecting the fact that the electrostatic and xenon ion drag forces exceed the barium ion pressure gradient force by about one order of magnitude. The ion flux to the emitter peaks at the downstream end, even in case 2 , in which barium flow from the insert is inhibited to model the effect of tungsten deposits.

The calculated neutral barium partial pressure near the emitter is compared to the equilibrium barium pressure over the reaction zone in Figs. 14(a) and 14(b). In both cases the equilibrium vapor pressure profile follows the cathode temperature profile, increasing monotonically in the downstream direction. The axial neutral pressure distribution first rises in the downstream direction, then drops where the electron density and temperature peak, due to ionization. The neutral pressure rises again near the orifice plate in a small recircu- 


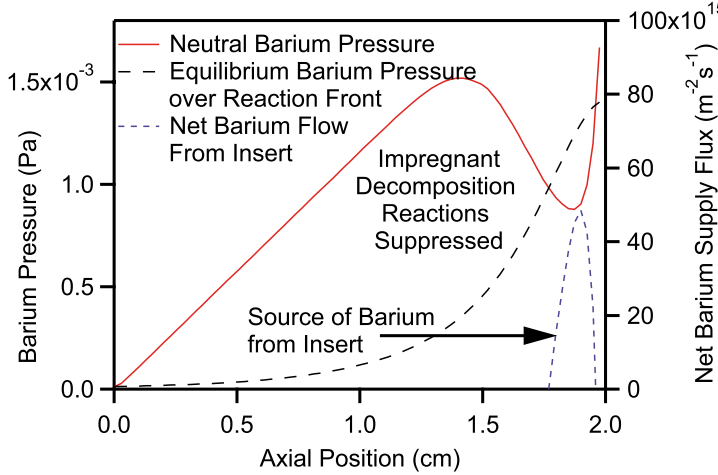

(a) Neutral barium pressure, equlibrium vapor pressure and net barium supply rate for Case 1 .

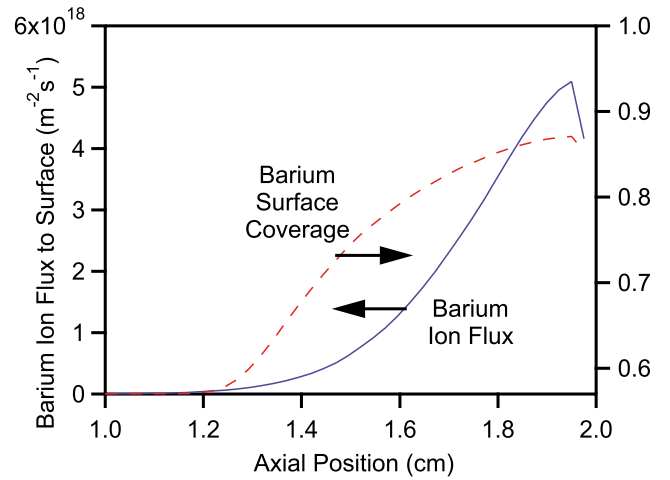

(c) Barium ion flux and surface coverage for Case 1 .

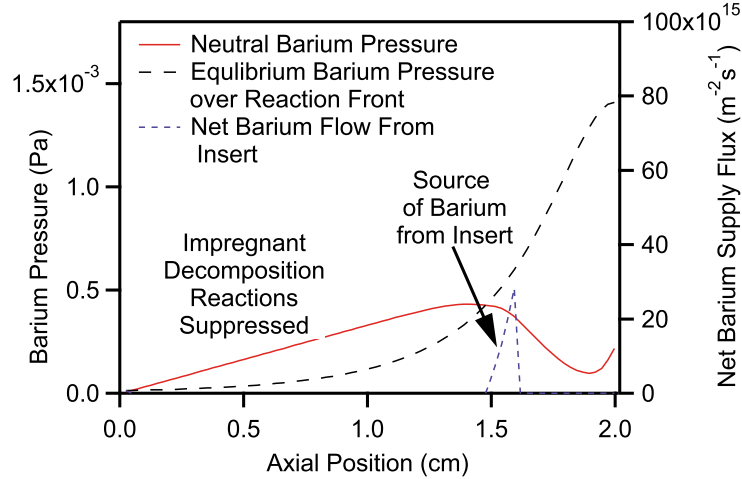

(b) Neutral barium pressure, equlibrium vapor pressure and net barium supply rate for Case 2 .

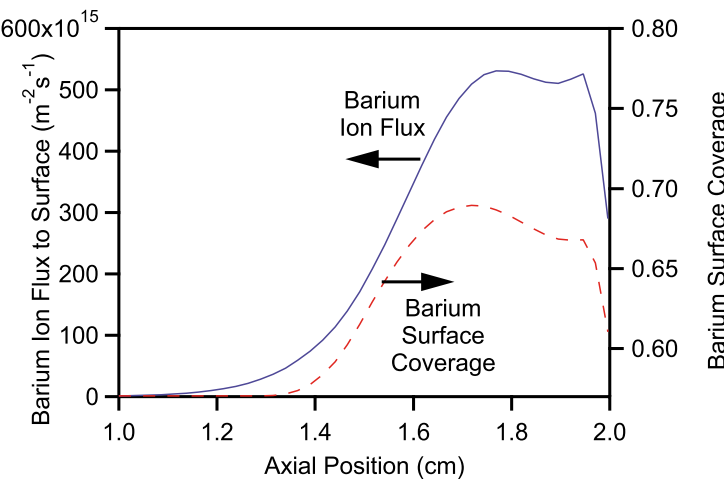

(d) Barium ion flux and surface coverage for Case 2.

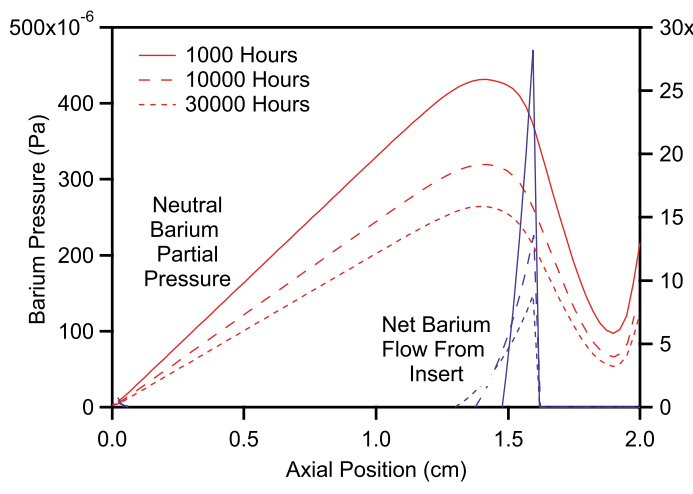

(e) Net barium flow from the insert and resulting neutral pressure as a function of operating time.

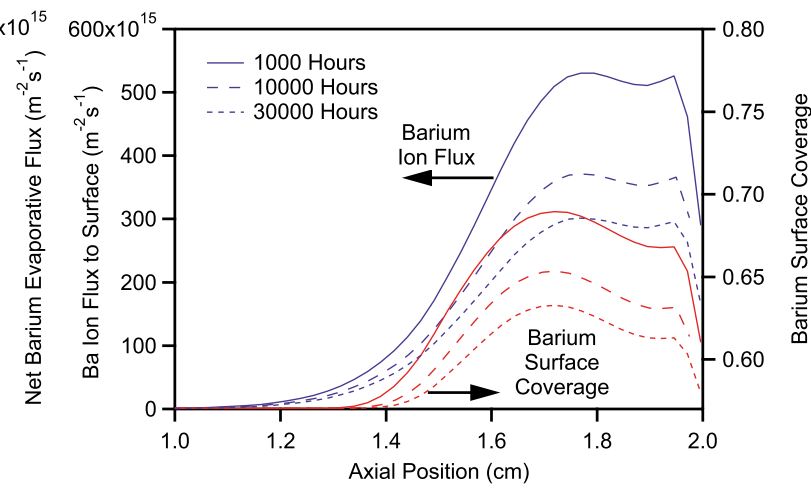

(f) Barium ion flux and equilibrium surface coverage as a function of operating time.

FIG. 14. (Color online) Axial distributions of neutral barium pressure, equilibrium vapor pressure, net barium supply rate from the insert interior, and the resulting ion flux and equilibrium surface coverage.

lation zone that is generated in the corner. The ambient barium pressure exceeds the equilibrium vapor pressure over most of the insert length, suppressing the reactions in the interior of the porous tungsten dispenser. In case 1 , barium is supplied from a region only about $2.5 \mathrm{~mm}$ long at the downstream end of the insert. In case 2, barium emission in this zone is inhibited, but the neutral pressure is lower by about a factor of 4, so a small region just upstream of this zone supplies barium (at a rate about four times lower than in case 1).

The axial distribution of the barium ion flux to the emitter is plotted in Figs. 14(c) and 14(d). For case 1, the ion flux peaks at the downstream end and has a maximum value of
$5 \times 10^{18} \mathrm{~m}^{-2} \mathrm{~s}^{-1}$. For case 2 , the ion flux distribution is broader and the peak value is about an order of magnitude lower.

\section{E. Surface coverage of barium due to transport in the gas phase}

The barium surface coverage that can be maintained with barium resupply from the gas phase can be estimated using a surface kinetics model. If we neglect the contribution from surface diffusion out of the pores in the tungsten and 
assume there are no weakly bound precursor states for adsorbed barium, the time rate of change of barium atom density on the surface can be expressed as

$$
N_{0} \frac{\partial \theta}{\partial t}=\left(\Gamma_{\mathrm{Ba}}+\Gamma_{\mathrm{Ba}^{+}}\right)(1-\theta)-\Gamma_{d} .
$$

In this expression, $N_{0}=5 \times 10^{18} \mathrm{~m}^{-2}$ is the number density of adsorption sites for barium on tungsten, ${ }^{24} \theta$ is the coverage, or fraction of these sites that are occupied, and $\Gamma_{\mathrm{Ba}}$ $=n_{\mathrm{Ba}} u_{t, \mathrm{Ba}}$ is the flux of barium atoms to the surface at the thermal velocity $u_{t, \mathrm{Ba}} \cdot \Gamma_{\mathrm{Ba}^{+}}=n_{\mathrm{Ba}+} u_{B}$ is the flux of ions, which enters the cathode sheath at the Bohm velocity $u_{B}$ $=\sqrt{k T_{e} / m_{\mathrm{Ba}}}$. The sticking coefficient of barium on tungsten is assumed to be unity, so the probability that impinging barium particles will adsorb in a given area on the surface is proportional to the fraction of open adsorption sites in that area, $(1-\theta)$.

The barium desorption rate $\Gamma_{d}$ can be described by an Arrhenius-type relationship, $\Gamma_{d}\left(T_{c}, \theta\right)$ $=N_{0} \nu_{d}(\theta) \exp \left[e E_{d}(\theta) / k T_{c}\right]$, where $e$ is the electron charge in coulombs. The desorption energy $E_{d}$ and pre-exponential factor $\nu_{d}$ are both strong functions of the coverage $\theta$ because of adatom interactions. At high values of $\theta$, interactions between neighboring adatoms reduce the binding forces, making it easier for adatoms to thermally desorb. This can be modeled semiempirically assuming a constant desorption energy and a pre-exponential factor that varies as $\theta^{5}$. Using desorption data for barium on oxidized tungsten, ${ }^{24}$ an expression for the desorption flux in this form can be derived,

$$
\Gamma_{d}=1.05 \times 10^{25} \theta^{5} \exp \left(-2.06 e / k T_{c}\right) .
$$

Under steady state conditions, the equilibrium surface coverage is given by a balance between the supply rate from the gas phase and loss by desorption,

$$
\frac{1-\theta}{\theta^{5}}=\frac{1.05 \times 10^{25} \exp \left(-2.06 e / k T_{c}\right)}{n_{\mathrm{Ba}} u_{t, \mathrm{Ba}}+n_{\mathrm{Ba}+} u_{B}} .
$$

For the conditions described here, the ion flux to the emitter is much higher than the neutral flux. The surface coverage corresponding to the calculated ion flux for the measured insert temperature distribution is plotted in Figs. 14(c) and 14(d). For case 1, the ion flux is so high at the downstream end that the coverage is close to 1 . This is a conservative calculation, because it ignores the contribution from surface diffusion out of the pores. For case 2, there is no contribution from the pores in this region and even though the ion flux is an order of magnitude lower, the coverage is still greater than 0.65 in the electron emission zone. This coverage is comparable to what is maintained in vacuum cathodes by pore flow alone. ${ }^{24}$ The coverage values for these two cases are not very different despite large differences in the ion flux because of the strong dependence of the desorption flux on coverage.

The results of simulations for case 2 with operating times of 1000, 10 000, and $30000 \mathrm{~h}$ are plotted in Figs. 14(e) and 14(f). Figure 14(e) shows that the neutral barium pressure drops as the barium flow from the insert is reduced by depletion (greater flow resistance as the reaction front recedes into the insert). The region over which barium can be produced broadens slightly as the barium partial pressure drops relative to the equilibrium vapor pressure, which is constant in this series of simulations because the cathode temperature is held constant. This broadening compensates to some extent for the barium depletion; more of the insert surface can supply barium as the production rate drops.

Figure 14(f) demonstrates again that the surface coverage is relatively insensitive to changes in ion flux. The ion flux drops almost a factor of 2 , roughly in proportion to the decreases in barium flow and neutral pressure, but the surface coverage drops only about $10 \%$. The coverage levels even after $30000 \mathrm{~h}$ of operation with no barium supply under the electron emission zone are still adequate to maintain high current densities.

\section{CONCLUSIONS}

The insert analyses and modeling resulted in three main conclusions that have significant implications for hollow cathode lifetime. First, tungsten erosion and subsequent redeposition in the emission zone result in the formation of a dense tungsten shell with reduced porosity. This deposit inhibits barium flow from the interior and therefore suppresses the reaction, resulting in very little barium depletion under the primary electron emission zone. Despite this, hollow cathodes appear to function correctly for very long lifetimes.

The barium transport model shows that barium in the downstream region can be very effectively supplied through the gas phase and will maintain adequate surface coverage in the emission zone for lifetimes of the order of those achieved in long duration tests. The key difference between hollow cathode gas discharges and vacuum dispenser cathodes is that hollow cathodes make much more efficient use of the barium supplied by the insert. Neutral barium from the insert is ionized in the intense xenon plasma and the combination of the electric field and the drag from the xenon ion flow results in a high barium ion flux to the emitter surface. The ions are neutralized on the surface and return to the discharge to be ionized again. Because the upstream boundary is the only effective sink for barium (except under conditions in which barium can accumulate on the cathode surface; in the presence of oxidizing impurities for instance) a high barium partial pressure builds up at the downstream end.

The high barium partial pressure appears to suppress impregnant reduction reactions over most of the insert length. Barium is evidently supplied only from a narrow region where ionization reduces the neutral density below the equilibrium vapor pressure. This result is the most surprising and perhaps the most uncertain because it relies on the relative values of thermochemical properties which are not well known. A careful examination of the depletion profile along hollow cathodes from long duration wear tests could help confirm this conclusion. This is another example of selfregulation in hollow cathodes-the barium production rate increases until the pressure builds up sufficiently to suppress reactions over some length of the insert. This suggests that barium located upstream of the electron emission site is not wasted, but is critical to the operation of the discharge through gas phase resupply and, in fact, much of this supply 
is saved until it is needed. These improvements in understanding of barium transport processes indicate that models of cathode failure due to barium depletion that are based on vacuum dispenser cathode data are likely to be extremely conservative.

\section{ACKNOWLEDGMENTS}

The authors would like to thank Al Owens, Ray Swindlehurst, and Ron Watkins for their assistance in preparing the test facility and Ron Ruiz and Jim Kulleck for their contributions in electron microscopy. The research described in this paper was carried out by the Jet Propulsion Laboratory, California Institute of Technology, under a contract with the National Aeronautics and Space Administration.

${ }^{1}$ D. Oh, 41st Joint Propulsion Conference, Sacramento, CA, 2006, Paper No. AIAA-2006-4270.

${ }^{2}$ A. Shroff, P. Palluel, and J. Tonnerre, Appl. Surf. Sci. 8, 36 (1981).

${ }^{3}$ J. Polk, J. Anderson, J. Brophy, V. Rawlin, M. Patterson, and J. Sovey, 35th Joint Propulsion Conference, Los Angeles, CA, 1999, Paper No. AIAA-99-2446.

${ }^{4}$ A. Sengupta, J. Brophy, and K. Goodfellow, 40th Joint Propulsion Conference, Fort Lauderdale, FL, 2004, Paper No. AIAA-2004-4558.

${ }^{5}$ A. Sengupta, 29th International Electric Propulsion Conference, Princeton, NJ, 2005, Paper No. iEPC 2005-026.

${ }^{6}$ I. Mikellides, I. Katz, D. Goebel, and K. Jameson, 41st Joint Propulsion Conference, Sacramento, CA, 2006, Paper No. AIAA-2006-5151.

${ }^{7}$ K. Jameson, D. Goebel, I. Mikellides, and R. Watkins, 41st Joint Propul- sion Conference, Sacramento, CA, 2006, Paper No. AIAA-2006-4490.

${ }^{8}$ J. Polk, 41st Joint Propulsion Conference, Sacramento, CA, 2006, Paper No. AIAA-2006-5153.

${ }^{9}$ J. Polk, 42nd Joint Propulsion Conference, Cincinnati, OH, 2007, Paper No. AIAA-2007-5191.

${ }^{10}$ A. Capece, J. Polk, and J. Shepherd, Space Propulsion 2008 Conference, Heraklion, Greece, 2008.

${ }^{11}$ T. Sarver-Verhey, 34th Joint Propulsion Conference, Cleveland, OH, 1998, Paper No. AIAA-98-3482.

${ }^{12}$ T. Verhey, 27th Joint Propulsion Conference, Sacramento, CA, 1991, Paper No. AIAA-91-2123.

${ }^{13}$ J. Brophy, Rev. Sci. Instrum. 73, 1071 (2002).

${ }^{14}$ J. Polk, D. Brinza, R. Kakuda, J. Brophy, I. Katz, J. Anderson, V. Rawlin, M. Patterson, J. Sovey, and J. Hamley, 27th International Electric Propulsion Conference, Pasadena, CA, 2001, Paper No. iEPC-01-075.

${ }^{15}$ I. Rudwan, N. Wallace, M. Coletti, and S. B. Gabriel, 30th International Electric Propulsion Conference, Florence, Italy, 2007, Paper No. iEPC 2007-256.

${ }^{16}$ G. Gärtner, P. Geittner, D. Raasch, and D. Wiechert, Appl. Surf. Sci. 146, 22 (1999).

${ }^{17}$ T. G. Walker, K. Bonin, and W. Happer, J. Chem. Phys. 87, 660 (1987).

${ }^{18}$ J. Dettmann and F. Karstensen, J. Phys. B 15, 287 (1982).

${ }^{19}$ E. Rittner, W. Rutledge, and R. Ahlert, J. Appl. Phys. 28, 1468 (1957).

${ }^{20}$ P. Palluel and A. Schroff, J. Appl. Phys. 51, 2894 (1980).

${ }^{21}$ I. Mikellides, I. Katz, D. Goebel, and J. Polk, J. Appl. Phys. 98, 113303 (2005).

${ }^{22}$ I. Mikellides, I. Katz, D. Goebel, and J. Polk, 41st Joint Propulsion Conference, Tucson, AZ, 2005, Paper No. AIAA-2005-4234.

${ }^{23}$ I. Mikellides, I. Katz, D. Goebel, J. Polk, and K. Jameson, Phys. Plasmas 13, 063504 (2006).

${ }^{24}$ R. Forman, J. Appl. Phys. 47, 5272 (1976). 